\title{
Artroplastia Reversa em um Caso de Reabordagem de Cisto Ósseo em Úmero Proximal
}

\author{
Reverse Arthroplasty in a Case of Bone Cyst Rebounding in a \\ Proximal Humber
}

Lucas Fraga ${ }^{1 *}$. Rogério Barros ${ }^{1}$, Roberto Maia ${ }^{1}$, Marcus Santos ${ }^{1}$, Rodrigo Martins ${ }^{1}$ ${ }^{1}$ Serviço de Ortopedia do Hospital Santa Izabel; Salvador, Bahia, Brasil

Correspondence addresses:

Dr. Lucas Fraga

fragacunha@gmail.com

Received: December 20, 2019

Revised: January 24, 2020

Accepted: January 30, 2020

Published: March 31, 2020

Data Availability Statement: All relevant data are within the paper and its Supporting Information files.

Funding: This work was the result of author's initiative. There was no support of research or publication funds.

Competing interests: The author has declared that no conflit of interests exists.

\section{Copyright}

(C) 2020 by Santa Casa de Misericórdia da Bahia.

All rights reserved.

ISSN: 2526-5563
Os cistos ósseos simples são, em regra, assintomáticos e encontrados incidentalmente, embora possam causar dor, edema, rigidez da articulação adjacente ou estarem associados a um quadro de fratura patológica. A curetagem é o modo mais comum de tratamento. O defeito ósseo após curetagem deve ser preenchido com enxertos ósseos ou substitutos, como hidroxiapatita, fosfato tricálcico e cimento. Entretanto, a falha no tratamento pode ser devastadora para o ombro, em decorrência da perda biomecânica da área. Assim, a reconstrução do úmero proximal, após a ressecção do cisto, é um grande desafio para o cirurgião ortopédico. Este caso relata a colocação de uma prótese reversa de ombro (PRO), realizada após a falha do tratamento de um cisto ósseo simples, como uma solução eficiente para a reparação do problema.

Palavras-chave: Cisto Ósseo; Defeito Ósseo; Prótese Reversa de Ombro.

Simple bone cyst are commonly asymptomatic and incidentally found, although they can cause pain, edema, stiffness of the adjacent joint or be associated with a pathological fracture. Curettage is the most common way of treating simple bone cysts. The bone defect after curettage must be filled with bone grafts or substitutes such as hydroxyapatite, tricalcium phosphate and cement. However, treatment failure can be devastating for the shoulder, due to the biomechanical loss of the area. Thus, the reconstruction of the proximal humerus after resection of the cyst is a major challenge for the orthopedic surgeon. This case reports the placement of a reverse shoulder prosthesis (PRO), performed after the failure of the treatment of a simple bone cyst, as an efficient solution to the problems related to the failure of treatment for the simple bone cyst.

Keywords: Bone Cyst; Bone defect; Reverse Shoulder Prosthesis.

\section{Introdução}

O cisto ósseo simples é um tumor benigno intramedular, sendo mais comum em esqueletos imaturos, principalmente em crianças ${ }^{1-3}$. Tal lesão possui origem metafisária e cresce para longe da fise, com afinamento cortical e expansão mínima. Os cistos ósseos simples são, em regra, assintomáticos e encontrados incidentalmente, embora possam ensejar dor, edema, rigidez da articulação adjacente ou estarem associados a um quadro de fratura patológica. 
A curetagem é o modo mais comum de tratamento de tumores ósseos benignos e de lesões líticas benignas, de maneira que o defeito ósseo, após a curetagem de tumores benignos, deve ser preenchido com enxertos ósseos ou substitutos, como hidroxiapatita, fosfato tricálcico e cimento. A falha nesse tratamento pode ser devastadora para o ombro, em decorrência da perda da singular biomecânica da área, que a caracteriza como a articulação com maior arco de movimento no corpo humano.

Nesse sentido, a reconstrução do úmero proximal, após a ressecção tumoral, continua sendo um grande desafio para o cirurgião ortopédico. A funcionalidade do ombro depende da estabilização complexa dos sistemas estático e dinâmico, incluindo os tecidos moles periarticulares, que são frequentemente sacrificados durante a ressecção, para que se obtenham margens livres de tumor, em caso de lesões malignas. Como consequência, a função articular é prejudicada por uma limitação de amplitude de movimento e uma instabilidade aumentada do ombro.

O objetivo desse trabalho é apresentar o resultado da colocação de uma prótese reversa de ombro (PRO), realizada após a falha do tratamento de um cisto ósseo simples, com perda significativa do estoque ósseo proximal do úmero e do manguito rotador.

\section{Relato de Caso}

Paciente do sexo feminino, 62 anos, apresentava histórico de dor em ombro esquerdo, principalmente à noite, irradiando para a face lateral do braço, com oito meses de evolução, sem trauma. Realizados exames de radiografia, tomografia e ressonância magnética, foi diagnosticado um cisto ósseo simples e uma ruptura parcial do supraespinhal, optando a paciente, em conjunto com seu médico assistente, pela realização de uma cirurgia de ressecção, preenchimento com cimento ósseo e a fixação com placa e parafusos (Figura 1).
Figura 1. Cirurgia de ressecção e preenchimento com cimento ósseo.
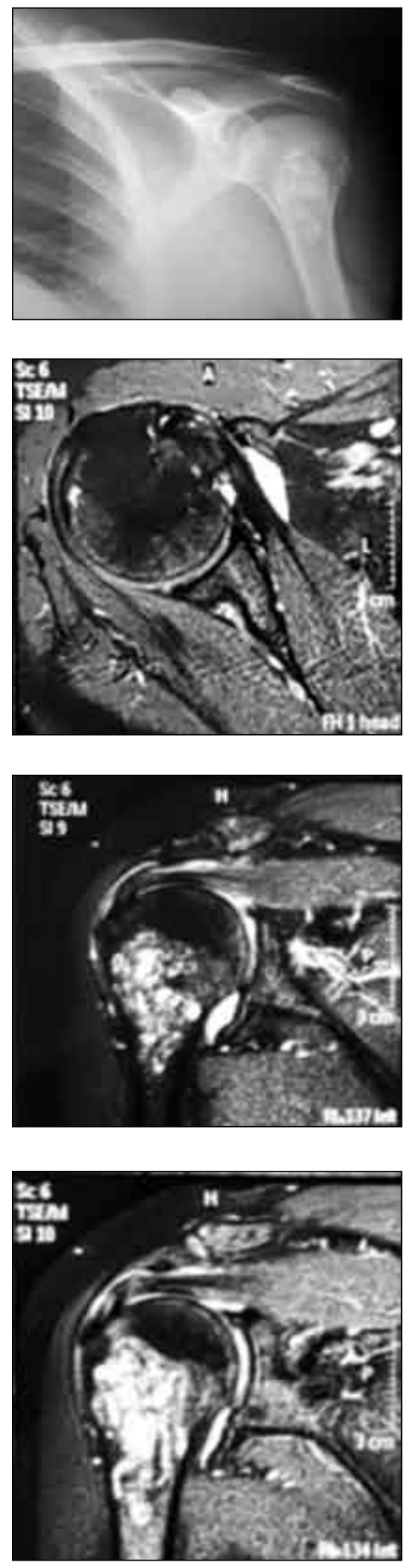
A recuperação ocorreu sem intercorrências até a segunda semana subsequente ao procedimento, quando a paciente relatou sentir dor intensa e incapacidade de elevar o braço, após a realização de sessões de fisioterapia, além de apresentar hiperemia em face lateral de ombro. Os exames de radiografia e de tomografia computadorizada revelaram uma perda da redução da cabeça umeral, com penetração de parafusos proximais. Os exames físicos e a ultrassonografia, por sua vez, indicaram uma lesão do manguito, manifestada pela elevação anterior de zero grau, que se repetiu em abdução, rotação interna e externa, enquanto que os testes laboratoriais suscitaram a possibilidade de infecção de sítio cirúrgico.

Para tratamento da suposta infecção, foi realizado um extenso debridamento. No intraoperatório, foi ratificada a existência de lesãomaciçadomanguitorotador(supraespinhal, infraespinhal, subescapular), sendo realizada a retirada de material síntese e de cimento e a ressecção proximal de úmero. Oito amostras foram encaminhadas para cultura, optando-se, ao final, pela colocação de espaçador de cimento com antibiótico (Figura 2).
Após a manutenção de baixos valores de provas inflamatórias, resultado de cultura negativo e a manutenção de hiperemia, a paciente persistiu com dor e elevação anterior de zero grau. A reação de hipersensibilidade ao material da placa foi aventada como diagnóstico diferencial e confirmada pelo respectivo teste.

Ao fim do ciclo de seis semanas de antibiótico empírico, o procedimento definitivo foi realizado por meio de acesso delto peitoral, dissecção por planos, retirada de espaçador, coleta de novas culturas e preparação de diáfise umeral com fresagem cuidadosa. Foram executadas ainda capsulotomia e fresagem de glenoide, com o objetivo de aumentar a tensão de partes moles, em razão da instabilidade inerente à perda de estoque ósseo do úmero proximal, escolhida a posição do peg central com inferiorização e inclinação aproximada de $10^{\circ}$, e a placa central fixada com 4 (quatro) parafusos, optando-se por uma glenosfera de $42 \mathrm{~mm}$, componente de polietileno com aumento de $3 \mathrm{~mm}$. Os testes para estabilidade da prótese não indicaram sinais de instabilidade, tendo sido manipulada $1 \mathrm{~g}$ de vancomicina em ferida cirúrgica e feito uma sutura por planos e curativo.

Figura 2. Inserção de espaçador de cimento com antibiótico.

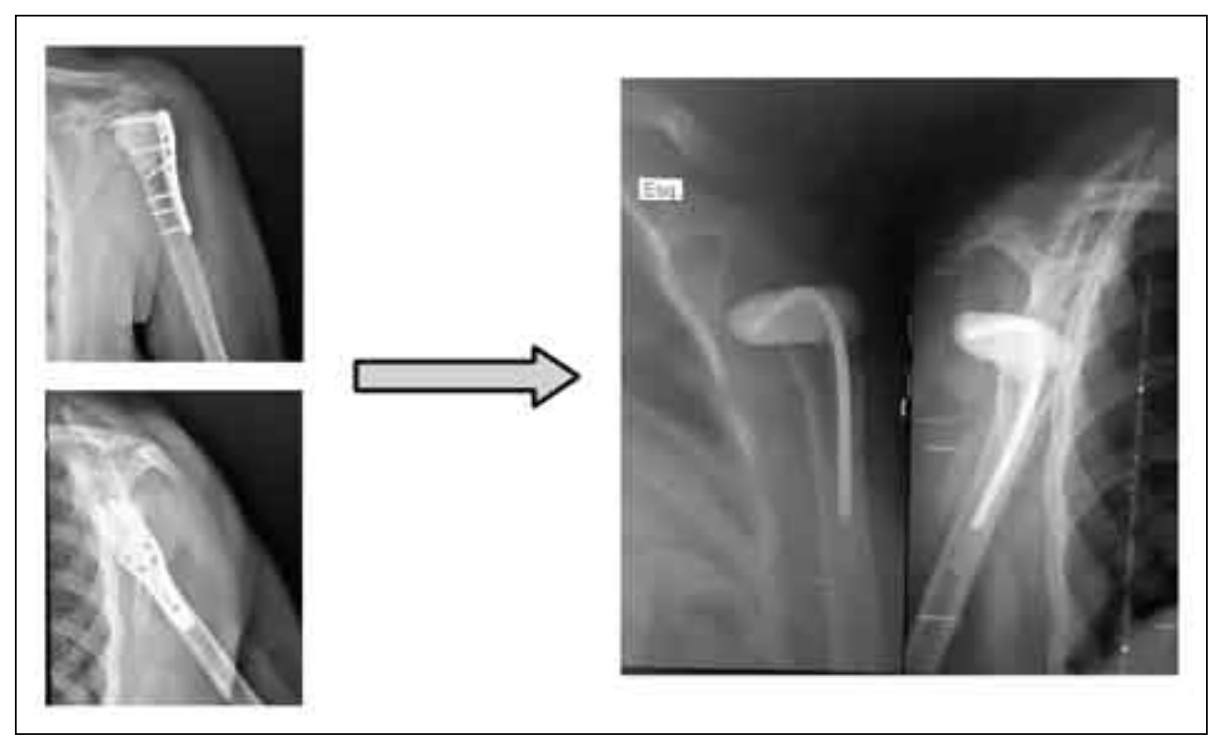


Figura 3. Ganho de arco de movimento com quatro semanas.
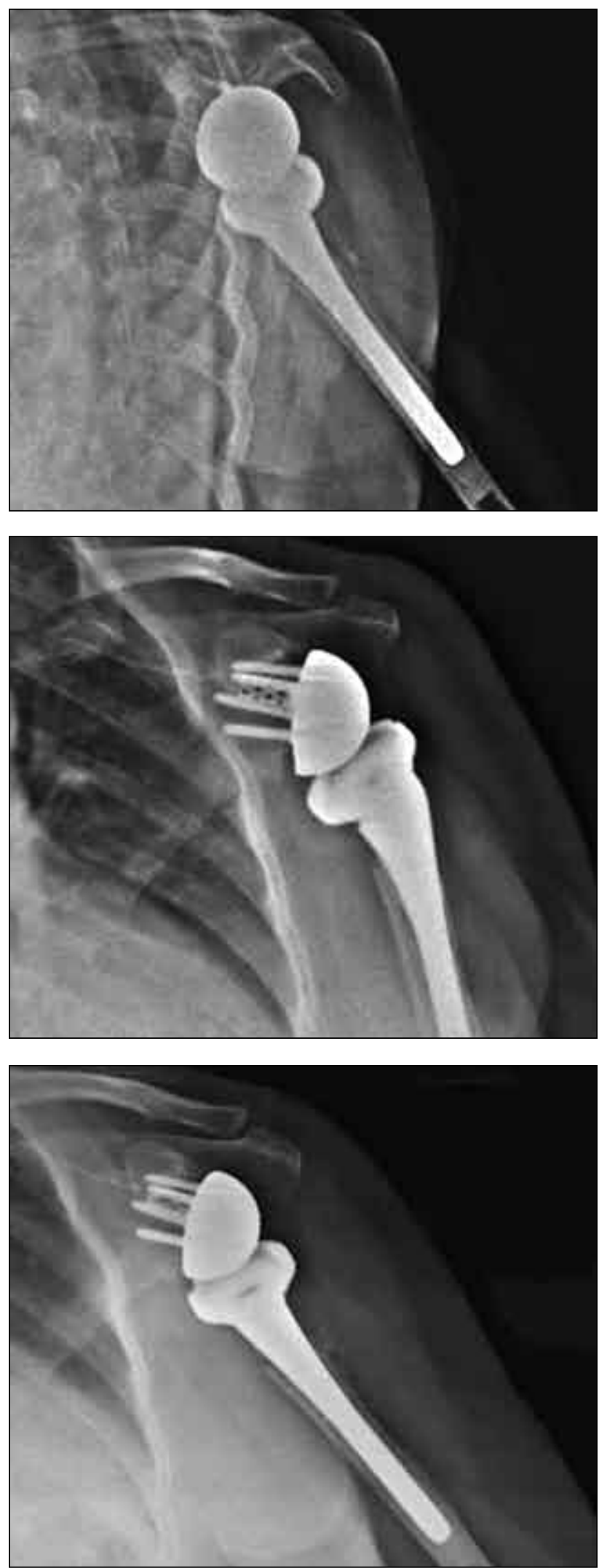

A paciente manteve o uso de tipoia de abdução por quatro semanas, seguida de duas semanas de tipoia simples, iniciando o ganho de arco de movimento com quatro semanas, conforme protocolo do serviço (Figura 3).

\section{Discussão}

Diferentes opções de tratamento, após a remoção do úmero proximal, têm sido descritas, incluindo endoprótese convencional, hastes umerais, aloenxerto osteoarticular e artrodese. $\mathrm{O}$ resultado funcional depende de diversos fatores, como a ressecção do manguito rotador, a possibilidade de preservação das inserções dos deltoides, latissimus dorsi e músculo peitoral no úmero, a preservação da função do nervo axilar e a verificação de ressecamento (ou não) em parte da escápula. ${ }^{4-6}$

Grammont foi o idealizador da prótese reversa do ombro, em 1970, como uma solução para a artropatia do manguito rotador (patologia em que o manguito rotador não é funcional) e a hemiartroplastia para falha no tratamento de fraturas do úmero proximal. ${ }^{2,3}$ Ao desenvolver uma prótese semiconstricta e com um centro de rotação medializado, permitiuse um ponto de apoio estável para o ombro, na ausência do manguito rotador, e um braço de alavanca aumentado do deltoide, ampliando sua capacidade no arco de movimento. Ao longo dos anos, o citado tratamento evoluiu, ${ }^{7}$ como se pode observar em autores como Bonnevialle e colegas, ${ }^{8}$ que apresentaram resultados promissores em seu estudo sobre o uso da PRO, principalmente após a ressecção de tumores malignos do úmero proximal. ${ }^{2,5}$

Com efeito, o uso desse procedimento em cirurgias de revisão de próteses aumentou, sendo os resultados satisfatórios, como demonstrado por Sanchez-Sotelo e colegas, ${ }^{9}$ ilustrando $73 \%$ de sucesso após cirurgias de revisão.

A perda de estoque ósseo no úmero proximal em tumor ósseo também foi abordada por Chacon e colegas, ${ }^{10}$ que usaram um composto 
de aloenxerto PRO em 25 e 21 pacientes, submetidos a um procedimento de revisão em decorrência da perda óssea após artroplastia de ombro. Os autores removeram entre 3,5 $\mathrm{cm}$ e $15 \mathrm{~cm}$ do úmero proximal e, em seguida, implantaram uma PRO, com uso de aloenxerto, fixado ao úmero restante por fios de cerclagem. Tal procedimento resultou em uma função melhorada do ombro na maioria dos pacientes, que foram acompanhados por, pelo menos, dois anos sem afrouxamento protético. O êxito do tratamento foi justificado pela redução do braço da alavanca, ocasionada pelo uso do aloenxerto, o que, teoricamente, reduziu o risco de afrouxamento.

Kaa e colegas, ${ }^{11}$ por sua vez, avaliaram dez pacientes com prótese reversa de ombro, sem o uso de aloenxerto para tumores proximais do úmero, pelo seguimento médio de tempo de quarenta e seis meses. Foi alcançada uma abdução media $78^{\circ}$, uma elevação anterior de $98^{\circ}$ (resultado semelhante ao obtido no presente estudo), elevação ativa anterior de $118^{\circ}$, abdução de $90^{\circ}$, rotação externa de $15^{\circ}$, rotação interna ao nível de T12, escore de Constant com 62 pontos, o que, comparado com outras análises, indica um resultado satisfatório. Entretanto, não foi encontrado, na literatura, o uso da PRO para falha de tratamento do cisto ósseo simples, para que fosse realizada a comparação dos resultados alcançados in casu com uma série de casos mais extensa.

Desse modo, verifica-se que a prótese reversa apresenta-se como uma solução eficiente para esse nicho de paciente e, simultaneamente, como uma opção incomum para as hipóteses de falha do tratamento cirúrgico de tumores benignos do úmero proximal.

\section{Referências}

1. Szendröi M, Sim FH. Color Atlas of Clinical Orthopedics. 2009:209-229.

2. Enneking WF. Musculoskeletal Tumor Surgery. Volume 1. New York, Churchill Livingstone; 1983; 87-89.

3. Teoh KH, Watts AC, Chee YH, Reid R, Porter DE. Predictive factors for recurrence of simple bone cyst of the proximal humerus. J OrthopSurg (Hong Kong). 2010;18:215-9.

4. Ghosh TK, Banik R, Bhattacharjya B. Evaluation of curettage, synthetic bone grafting and fixation with implant in benign osteolytic and cystic lesions of long bones in children and young adults. Int $\mathrm{J}$ Res Orthop2020;6:170-7.

5. Kassab M, Dumaine V, Babinet A, Ouaknine M, Tomeno B, Anract P. Twenty Nine Shoulder Reconstructions after resection of the proximal humerus for neoplasm with a mean 7 year follow-up. Rev ChirOrthopReparatriceAppar Mot. 2005;91(1):15e23.

6. Wittig JC, Bickels J, Kellar-Graney KL, Kim FH, Malawer MM. Osteosarcoma of the proximal humerus: long-term results with limb-sparing surgery. Clin Orthop Relat Res. 2002;397:156e176.

7. Grammont PM, Baulot E. The classic: delta shoulder prosthesis for rotator cuff rupture. Clin Orthop Relat Res. 1993;469(9):2424.

8. Bonnevialle $\mathrm{N}$, Mansat $\mathrm{P}$, Lebon J, Laffosse JM, Bonnevialle P. Reverse shoulder arthroplasty for malignant tumors of proximal humerus. J Shoulder Elbow Surg 2015;24:36-44. http://dx.doi.org/10. 1016/j.jse.2014.04.006

9. Sanchez-Sotelo J, Sperling J, Rowland C, Cofield R. Instability after shoulder arthroplasty: results of surgical treatment. J Bone JtSurg Am. 2003;85(A):622e631

10. Ariel Chacon, Nazeem Virani, Robert Shannon, Jonathan C. Levy, Derek Pupello, Mark Frankle J Bone Joint Surg Am. Revision arthroplasty with use of a reverse shoulder prosthesis-allograft composite. 2009 Jan;91(1):119-127. doi: 10.2106/JBJS.H.00094

11. Kaa AK, Jørgensen PH, Søjbjerg JO, Johannsen HV. Reverse shoulder replacement after resection of the proximal humerus for bone tumours. Bone Joint $\mathrm{J}$ 2013;95-B:1551-5. http://dx.doi.org/10.1302/0301620X. 95B11.31545 